Further Section

\title{
Neuropsychobiology 1977;3:257
}

\section{Author Index}

Ackenheil, M. 23 Agnoli, A. 42 Amdisen, A. 30, 153 Andraca, I. de 12 Ast, M. 144

Beckmann, H. 49 Belmaker, R.H. 105,250 Bernasconi, S. 35,135 Brambilla, F. 160 Brunetta, M. 160

Caroleị, A. 42 Cusumano, G. 35, 135

Dall'Olio, R. 1 Darling, S. 30,153 Deckard, B.S. 179

Ebstein, R.P. 105, 250

Farská, I. 120, 129 Feỉne, R. 105 Fink, M. 167 Foster, F.G. 167

Gandol:fi, O. 1 Glueck, B.C. 193 


\section{Henderson, A.J. 111}

Jacquy, J. 240 Jarvikik, L.F. 179 Jocquet, P. 240

Kadouch, R. 250 Kierkegaard-Hansen, A. Knorring, L. von 65 Krulịk, R. 120,129 Kupfer, D.J. 167

Lanng Nielsen, J. 30 Lhoas, J.P. 240 Lolalas, F. 12

Masturzo, P. 42 Matussek, N. 23 Meco, G. 42 Metzig, E. 144 Mirabile, Ch.S., jr. 193 Montanaro, N. 1 Murphy, D.L. 49

Noel, G. 240

Oreland, L. 65 
Paykel, E.S. 111 Pedersen, E.B. 30, 153 Peres, L. 250 Perris, C. 65 Piraux, A. 240 Pitblado, C, 193 Pollerí, A. 4253 Prokeš, J. 120, 129 Rimon, R. 105 Rolandï, E. 42 Römisch, P. 23 Rosenberg, S. 144

Sacchetti, E. 160 Saletu, B. 75 Siva Sankar, D.V. 234 Snider, S.R. 144 Spiker, D.G. 167 Strocchi, P. 1 Stroebel, Ch.F. 193 Tobin, D. 144

Valzelli, L. 35,135 Van Praag, H.M. 56

Wirz-Justice, A. 199 